

\title{
Solitonic characteristics of Airy beam nonlinear propagation
}

Thomas Bouchet, Nicolas Marsal, Marc Sciamanna, Delphine Wolfersberger

\section{To cite this version:}

Thomas Bouchet, Nicolas Marsal, Marc Sciamanna, Delphine Wolfersberger. Solitonic characteristics of Airy beam nonlinear propagation. Physical Review A: Atomic, molecular, and optical physics [1990-2015], 2018, 97, pp.051801(R). 10.1103/PhysRevA.97.051801 . hal-01879111

\section{HAL Id: hal-01879111 \\ https://hal-centralesupelec.archives-ouvertes.fr/hal-01879111}

Submitted on 30 Nov 2020

HAL is a multi-disciplinary open access archive for the deposit and dissemination of scientific research documents, whether they are published or not. The documents may come from teaching and research institutions in France or abroad, or from public or private research centers.
L'archive ouverte pluridisciplinaire HAL, est destinée au dépôt et à la diffusion de documents scientifiques de niveau recherche, publiés ou non, émanant des établissements d'enseignement et de recherche français ou étrangers, des laboratoires publics ou privés. 


\title{
Solitonic characteristics of Airy beam nonlinear propagation
}

\author{
Thomas Bouchet, Nicolas Marsal, Marc Sciamanna, and Delphine Wolfersberger \\ Chair in Photonics, LMOPS, CentraleSupélec, Université Paris-Saclay, F-57070 Metz, France \\ and Chair in Photonics, LMOPS, CentraleSupélec, Université de Lorraine, F-57070 Metz, France
}

(Received 27 September 2017; published 17 May 2018)

\begin{abstract}
We analyze the nonlinear propagation of a one-dimensional Airy beam. Under nonlinear focusing conditions, the Airy beam splits into a weak accelerating structure and a beam that has been named an "off-shooting soliton." Experimental measurements and numerical results related to the off-shooting Airy beam are compared to soliton theoretical profiles and a good agreement is found in terms of transverse shape, width, and amplitude. We identify the different parameters to generate an Airy beam off-shooting soliton and demonstrate that its profile is also preserved through propagation over long distances.
\end{abstract}

DOI: 10.1103/PhysRevA.97.051801

Dispersion-free Airy wave packets were first predicted by Berry and Balazs in the context of quantum mechanics [1]. By truncating the ideal Airy wave form, and applying it in optics, the first optical Airy beam was observed in 2007 [2]. Contrary to the ideal Airy solution in quantum mechanics, an optical Airy beam displays a finite energy but it retains a shape-preserving accelerating propagation over a finite distance and self-healing properties. Such unique features offer a large range of applications in a variety of domains, such as optical micromanipulation [3], plasma channel generation [4], all-optical routing [5,6], or light-sheet microscopy [7].

Recently, the self-trapping of Airy beams in biased nonlinear media has suggested interesting dynamics, such as solitonlike behaviors and interactions of co- and counterpropagating Airy beams [8-12]. In particular, under focusing nonlinear conditions, the Airy beam may split into a weak accelerating structure and a structure that has been named an "off-shooting soliton" (OSS) and that propagates along the medium without transverse acceleration [13]. The interactions between the photoinduced OSS and the accelerating beam have been studied recently [14], resulting in attraction, deflection, and tightening effects of the OSS and interesting analogies with gravitational lensing and tidal forces. The OSS has also been studied in the context of optical interconnects, offering more complex all-optical waveguiding possibilities than those achieved with Gaussian beams [6]. These two configurations suggest that the OSSs share properties that are typically attributed to spatial solitons.

In spite of these recent achievements, one question remains of interest: Is the Airy beam OSS a spatial soliton? To answer this question, it is worth reminding what defines a soliton. An optical soliton is a solitary wave packet that propagates at a constant velocity and maintains its shape due to nonlinear effects compensating dispersive effects. Particularly, spatial solitons are self-trapped beams of light that propagate while maintaining their shape due to the nonlinear effect compensating the diffraction of the propagating beam [15]. Optical solitons have been studied extensively for the past three decades in a variety of domains [16], in particular, in photorefractive media where they appear to be stable in one and two transverse dimensions [17].

In this Rapid Communication, we study and analyze both experimentally and theoretically the nonlinear propagation of a one-dimensional (1D) Airy beam in a $\mathrm{Sr}_{x} \mathrm{Ba}_{1-x} \mathrm{Nb}_{2} \mathrm{O}_{6}(\mathrm{SBN})$ photorefractive crystal. Under nonlinear focusing conditions, as expected, the Airy beam splits into a weak accelerating structure and an OSS. We demonstrate that the OSS matches with the theoretical soliton profile in terms of width and amplitude. We also prove that its profile remains unchanged during propagation. Finally, we analyze the OSS output position and width behavior versus its amplitude and show that its characteristics match the existence curve of the theoretical spatial soliton.

As depicted in Fig. 1(a), our experiment consists of propagating a one-dimensional Airy beam into a biased photorefractive SBN crystal with dimensions $5 \mathrm{~mm} \times 5 \mathrm{~mm} \times 2 \mathrm{~cm}$. The Airy beam is generated using a cubic phase modulation on a spatial light modulator [2]. We observe the profile of the Airy beam OSS at the output face of the crystal using a CCD camera. When a bias external electrical field is applied along the crystal $c$ axis, the optical Airy beam photoinduces a refractive index variation inside the crystal through the Pockels effect. For high enough nonlinearity strength, most of the beam turns into a so-called off-shooting soliton, while a small fraction of the power remains a self-accelerating linear packet $[13,14]$. Figures $1(\mathrm{~b})-1(\mathrm{~d})$ show the beam output captured on the camera with and without an external electric field applied. The experimental transverse profile of each OSS [Fig. 1(e)] is extracted from the brightest areas of Figs. 1(c) and 1(d). We can note that the OSS width and amplitude can be controlled by changing the voltage applied to the crystal. The analysis of each profile and their comparison to a theoretical soliton profile is done using the following theory described in Ref. [18] that was developed for solitons.

A one-dimensional steady-state bright screening soliton profile is described by the following reduced wave equation [18],

$$
\frac{d^{2} u}{d \xi^{2}}+\frac{u}{u_{0}^{2}} \ln \left(1+u_{0}^{2}\right)-\frac{u}{1+u^{2}}=0
$$




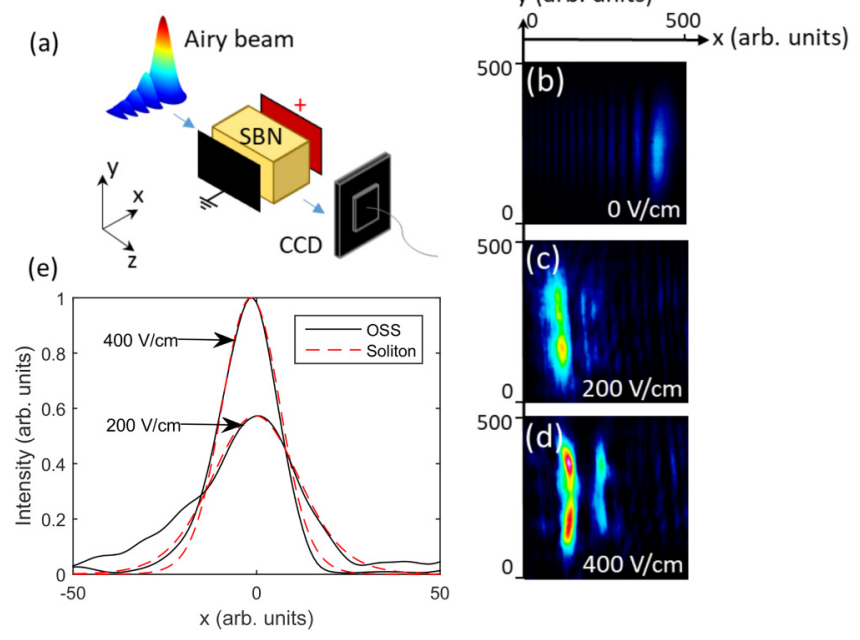

FIG. 1. (a) Schematic of the experimental setup: 1D Airy beam propagating in a 2 -cm-long SBN crystal $\left(x_{0}=10, a=0,1\right)$. (b) Observation of the Airy beam at the crystal output face on the camera with no electric field $E_{\text {ext }}$ applied. (c), (d) Observation of the OSS at the crystal output face on the CCD camera $\left(E_{\text {ext }}=V / l=200\right.$ and $400 \mathrm{~V} / \mathrm{cm}$ ). (e) Corresponding experimental Airy beam OSS profile extracted from the camera and theoretical classic soliton fit $\left(E_{\text {ext }}=200\right.$ or $400 \mathrm{~V} / \mathrm{cm}$ and $\left.P_{A}=50 \mu \mathrm{W}\right)$.

whose first integral is

$$
\frac{d u}{d \xi}=\left[\ln \left(1+u^{2}\right)-\left(u^{2} / u_{0}^{2}\right) \ln \left(1+u_{0}^{2}\right)\right]^{1 / 2}
$$

and where $u(\xi)$ is the soliton amplitude divided by the square root of the effective background intensity (defined as the sum of the background and dark intensities induced respectively by an external homogeneous illumination of the crystal and the intrinsic thermal excitation of charges inside the crystal), $\xi=x / d$ is the transverse coordinate normalized by $d=\left(k^{2} n_{b}^{2} r_{\text {eff }} V / l\right)^{-1 / 2}, u_{0}$ is the maximum amplitude of the soliton at $\xi=0, k=2 \pi n_{b} / \lambda$ is the wave vector, $n_{b}$ is the unperturbed refractive index, $\lambda$ is the wavelength, $r_{\mathrm{eff}}$ is the effective component of the electro-optic tensor, $V$ is the voltage applied onto the crystal, and $l$ is the crystal's width.

Equation (2) can be solved using a Runge-Kutta method in order to obtain the theoretical soliton profile. In practice, this means that for each different nonlinear condition corresponding to the $d$ criteria and maximum soliton amplitude $u_{0}$, we obtain a different soliton profile. Once $d$ and $u_{0}$ are determined from our experiment $\left(n_{b}=2.3, r_{\text {eff }}=235 \mathrm{pm} / \mathrm{V}\right.$, $V=100$ or $200 \mathrm{~V}$, and $l=0.5 \mathrm{~cm}$ ), we therefore have access to the corresponding theoretical soliton profile whose plot is superimposed in Fig. 1(e) with the transverse experimental profiles. We evidence that under a focusing condition, the Airy beam sheds an OSS whose profile matches the theoretical soliton profile.

Although the experimental profile of the OSS fits with the theoretical soliton profile, it is worth mentioning that experimentally we only image the output and input faces of the crystal due to the nonhomogeneous refractive index that alters the imaging system inside the medium. In order to observe the formation and evolution of the OSS inside the crystal versus different parameters (crystal length or beam intensity, for example), we use a numerical model to simulate the OSS propagation. In what follows, we numerically propagate a $1+1 \mathrm{D}$ Airy beam in a photorefractive medium. The model is similar to the one presented in Refs. [6,12,14,19]. The normalized nonlinear Schrödinger equation reads

$$
i \partial_{z} F+\partial_{x}^{2} F=\Gamma E_{0} F,
$$

where $F$ is the beam's electric field amplitude, $\Gamma=$ $\left(k n_{0} x_{0}\right)^{2} r_{\mathrm{eff}} E_{e}$ is the nonlinear photorefractive coupling strength, and $E_{e}$ is the external electric field. $E_{0}$ is the homogeneous part of the $x$ component of the photorefractive space-charge field normalized by the external electric field applied. The temporal evolution of the space-charge field $E_{0}$ is considered with saturable nonlinearity and calculated using a relaxation-type dynamic,

$$
\tau \partial_{t} E_{0}+E_{0}=-\frac{I}{1+I},
$$

where the relaxation time of the crystal $\tau$ is inversely proportional to the total intensity $\tau=\frac{\tau_{0}}{1+I}$, and $I=|F|^{2}$ is the intensity normalized by the effective background intensity.

The beam propagates along the $z$ axis, and has a truncated Airy beam transverse profile upon injection at $z=0$,

$$
F(x, z=0)=F_{0} \operatorname{Ai}\left(\frac{x}{x_{0}}\right) e^{a \frac{x}{x_{0}}},
$$

where $F_{0}$ is the total amplitude of the Airy beam, Ai is the Airy function, $x_{0}$ is the Airy beam's main lobe waist at $1 / e$ of its maximum intensity, and $a$ is the truncation parameter.

Based on this model, we numerically propagate a $1+1 \mathrm{D}$ Airy beam in a photorefractive medium using a beam propagation method within a temporal loop that recalculates the electric field inside the crystal at each time iteration. The study is done once steady state is reached (more than $50 \tau$ ).

Figure 2(a) shows the linear propagation at steady state of the 1D Airy beam along the $z$ axis of a photorefractive medium. Similar to Ref. [6] and in order to fit to the experiment presented here, the truncation and waist parameters are $x_{0}=10 \mu \mathrm{m}$ and $a=0.1$. As expected, we observe a curved trajectory for a $2-\mathrm{cm}$ propagation length inside the medium. (a)

(c)

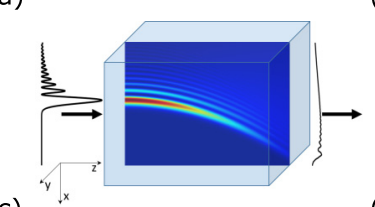

(b)


FIG. 2. Propagation of a 1D Airy beam in a 2-cm-long photorefractive crystal at steady state. (a) Linear propagation $(\Gamma=0)$. (b) Nonlinear propagation in the presence of an external electric field leading to an OSS ( $\Gamma=10, F_{0}=1.95, x_{0}=10 \mu \mathrm{m}, a=0.1$ ). (c), (d) Distribution of the refractive index change vs $x$ and $z$ at steady state. Arrows represent the Poynting vector. (c) $\Gamma=3$. (d) $\Gamma=10$. 
Moreover, our numerical simulation reproduces qualitatively well our experimental result since, similarly to the experiment in Fig. 1, when an external electric field is applied $(\Gamma>0)$, we numerically observe the shedding of an OSS from the original Airy beam [Fig. 2(b)]. It is worth noting that for a smaller apodization factor we observe several off-shooting beams in different directions of propagation (similar to Ref. [9] in Kerr media). In our study we decided to focus on the OSS which does not deviate from the propagation axis.

Figure 2(c) [respectively Fig. 2(d)] represents the photoinduced distribution of the refractive index change $\Delta n=$ $-\frac{n_{0}^{3}}{2} r_{\text {eff }} E_{s c}$ inside the crystal for $\Gamma=3$ (respectively $\Gamma=10$ ) versus $x$ and $z$. To gain further insight, we superimpose the time-averaged Poynting vector $\langle\vec{S}\rangle$ using the following equation from Refs. [20,21],

$$
\langle\vec{S}\rangle=i \omega \frac{\epsilon_{0}}{2}\left(F_{x} \frac{\partial F_{x}^{*}}{\partial x}-F_{x}^{*} \frac{\partial F_{x}}{\partial x}\right) \vec{u}_{x}+\omega k \epsilon_{0}|F|^{2} \vec{u}_{z},
$$

where $\epsilon_{0}$ is the vacuum permittivity and $\omega=c k$ with $c$ the speed of light. Increasing the nonlinearity $\Gamma$ leads to the creation of a photoinduced waveguide that modifies the beam's propagation. The arrows show how energy is transferred from the main lobe to the second lobe which is then transferred to the next lobes, hence explaining the creation of the OSS.

We now propose to compare the numerical OSS with the theoretical soliton and analyze its behavior versus $u_{0}$. We therefore link the two models using

$$
d=\frac{x_{0}}{\sqrt{\Gamma}}
$$

and fix the maximum amplitude of the Airy beam OSS as $u_{0}$. Then we can solve Eq. (2) and compare the different profiles.

Figure 3(a) shows the OSS numerical amplitude profile versus $x$ corresponding to Fig. 2(b) after a propagation of $2 \mathrm{~cm}$ at steady state. The amplitude does not reach the zero value in the tails of the solitonlike profile because the diffraction of the Airy beam multilobe structure creates residual lighting on the numerical simulation [Fig. 3(b)]. The OSS numerical


FIG. 3. (a) Numerical profile of the Airy beam OSS in Fig. 2(b) and corresponding theoretical soliton at $L=2 \mathrm{~cm}$. (b) Propagation of the OSS over long distances at steady state $\left(\Gamma=10, F_{0}=1.95\right.$, $\left.x_{0}=10 \mu \mathrm{m}, a=0.1, u_{0}=1.04\right)$.
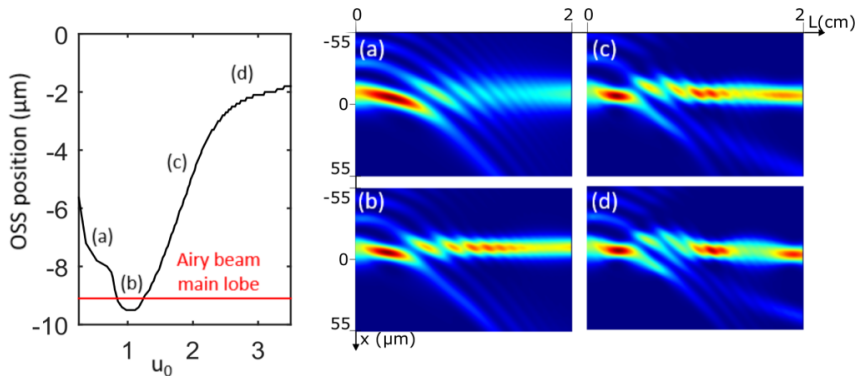

FIG. 4. Position of the Airy beam OSS as function of OSS maximum amplitude for $\Gamma=10, F_{0}=1-7.5$ or $u_{0}=0.25-3.5$, $x_{0}=10 \mu \mathrm{m}, a=0.1$, and $t_{f}=20 \tau_{0}$. (a)-(d) Corresponding numerical simulation inside the crystal for (a) $u_{0}=0.5$, (b) $u_{0}=1.04$, (c) $u_{0}=1.9$, (d) $u_{0}=2.9$.

profile shows a transverse profile that matches a solitonlike profile. By using the model from Ref. [18] [Eqs. (1) and (2)] and expression (7), we can link our numerical simulations with the theoretical soliton model. We draw the theoretical soliton profile that would propagate in the crystal under the same conditions as our numerical simulation. Figure 3(a) demonstrates that the theoretical soliton profile fits nicely with the numerical profile of the OSS.

Is the solitonlike profile of the OSS stable over long distances? To answer this question, we numerically propagate the OSS up to $12 \mathrm{~cm}$ inside the nonlinear medium (corresponding to more than 20 diffraction lengths). The profile illustrated in Fig. 3(b) shows a constant behavior over the 12 -cm propagation length. The maximum amplitude between the OSS at $2 \mathrm{~cm}$ and the one at $12 \mathrm{~cm}$ of propagation differs by only $1 \%$, unveiling its solitonlike nature.

We can now fairly consider the Airy beam OSS as a spatial soliton even after $2 \mathrm{~cm}$ of propagation inside the crystal. But as observed in Ref. [14], it is important to note that the output position of the OSS varies slightly with the intensity of the Airy beam sent into the nonlinear medium. Figure 4 shows at steady state the evolution of the OSS position at the output face of the crystal as a function of the off-shooting beam's maximum amplitude. This curve is drawn by increasing numerically the total amplitude of the input Airy beam $F$ from 1 to 7.5, which corresponds to the maximum amplitude $u_{0}$ of the OSS going from 0.25 to 3.5. We notice that the OSS position is correlated to different propagation behaviors. For $0.5<u_{0}<2$ [Figs. 4(a)-4(c)] the position of the OSS at steady state is close $\left(<5 \mu \mathrm{m},<x_{0} / 2\right)$ to the position of the Airy beam's main lobe at the input of the crystal (red line in Fig. 4). We also checked that the OSS beam profile corresponds to that of a theoretical soliton in this region. When $u_{0}>2$ [Fig. 4(d)], the OSS trajectory is no longer perpendicular to the input face of the crystal, the solitonic profile is lost, and we observe spatial breathing along the propagation axis. For Figs. 4(a) and 4(c) the trajectory of the OSS is not perpendicular to the input face of the crystal and the position of the OSS shifts along the $x$ axis over greater distances, whereas in the area close to the extremum of the curve [Fig. 4(b)], the position of the soliton no longer changes over greater distances.

Referring to Figs. 4(a)-4(d) we can observe variations of the intensity and width of the OSS at the output face 


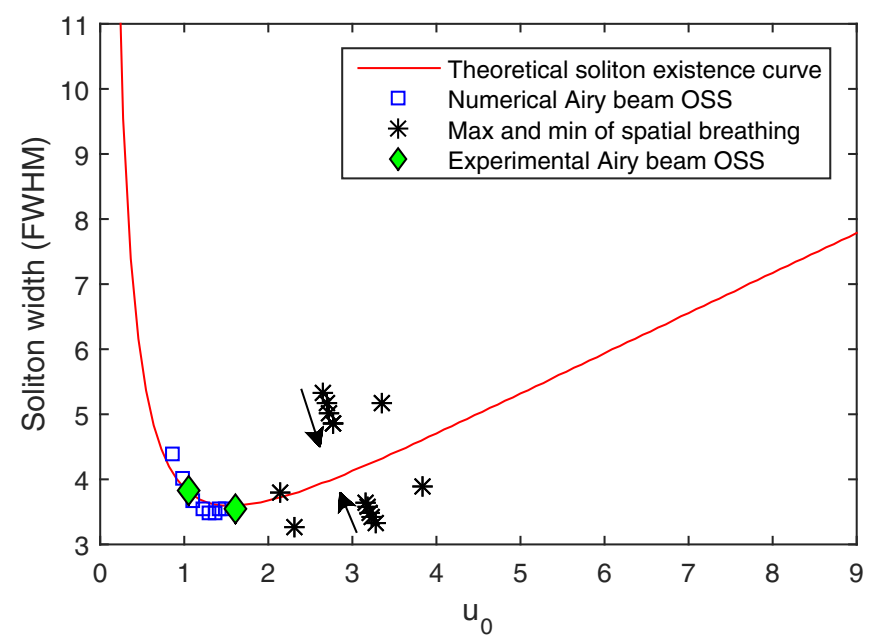

FIG. 5. Full width at half maximum (FWHM) in dimensionless units $(\xi)$ versus of soliton amplitude $\left(u_{0}\right)$. In red, the theoretical curve from Ref. [18]; in blue squares, the Airy beam OSS at 2-cm propagation from our numerical model; in black stars, the maximum and minimum of the observed spatial breathing; and the arrows show the evolution when propagating over greater distances. In green diamonds, the experimental OSS observed in Fig. 1.

of the medium. The theory of one-dimensional steady-state screening solitons in bulk material states that a relation exists between the width and the intensity of the soliton: Fig. 5 depicts the theoretical existence curve of the soliton full width at half maximum (FWHM) as a function of $u_{0}$ from Ref. [18]. The green diamonds represent the experimental Airy OSS seen in Fig. 1 for the two different applied electric fields (200 and $400 \mathrm{~V} / \mathrm{cm}$ ). These experimental points are on the existence curve and confirm that the obtained OSS is indeed a soliton. Also represented in this figure is the Airy beam off-shooting FWHM for different values of $u_{0}$, all selected close to the extrema in Fig. 4, and obtained by solely modifying the amplitude of the input Airy beams. The values of $u_{0}$ go from 0.8 to 1.5 and we measure the corresponding FWHM of the OSS. We see that our numerical OSS width and theoretical curve are in good agreement, further corroborating the similarity between OSS and a classical soliton. At greater values of $u_{0}$, where spatial breathing takes place, the maximum and minimum of the FWHM and corresponding $u_{0}$ are included in Fig. 5 as black stars. These points are on either side of the existence curve. Therefore, the beam's profile oscillates around the soliton profile. Furthermore, the amplitude and FWHM of theses spatial oscillations tend to converge towards the theoretical soliton existence curve over greater distances (arrows in Fig. 5).

The two experimental profiles are obtained using the same Airy beam but different applied electric fields on the crystal. Therefore, we can control the output width and amplitude of the OSS by changing the electric field applied on the crystal. For a given Airy beam input profile, the OSS fits with the theoretical plot for values of $u_{0}$ from 0.8 to 1.5 . The values of $u_{0}$ from 0.8 to 1.5 correspond to the extrema in position of the OSS as a function of $u_{0}$ (seen in Fig. 4). We conclude that the extrema in position versus $u_{0}$ indicates the area for which the OSS is closest to a theoretical soliton (blue squares in Fig. 5). Furthermore, since there is a quasilinear relation between the Airy beam amplitude and the OSS amplitude, we can also control the output OSS width and amplitude by changing the amplitude of the Airy beam. Therefore, unlike Gaussian beams, with one Airy beam we can create solitons of different widths when we change the nonlinearity in the crystal by modifying either the light intensity or the applied electric field.

In summary, we have evidenced the solitonic properties of Airy beam nonlinear propagation. Differently from Gaussian beams, the OSS coexists with a remaining self-accelerating structure. Experimental and numerical profiles of the OSS fit nicely with the theoretical soliton profile. Numerical simulations confirm that the OSS propagates invariably over great distances (more than 20 diffraction lengths). We observed that for fixed nonlinear conditions inside a photorefractive crystal, there exists a range of intensity values for which the OSS property is the closest to an ideal theoretical soliton. Our work motivates further studies of 2D Airy beams propagating in a photorefractive medium for testing the properties and the stability of the 2D OSS. Furthermore, since the OSS profile is quite similar to previously observed solitons such as those arising from Gaussian beams, our study is thought to be of interest for a large community and, in particular, those analyzing interactions and applications of spatial solitons.

We acknowledge the support of AIRBUS-GDI Simulation, Metz Métropole, Conseil Départemental de Moselle, Conseil Régional Grand-Est, Préfecture de Région Grand-Est, CentraleSupélec, Fondation Supélec and FEDER through the funding of the Chair in Photonics.
[1] M. V. Berry and N. L. Balazs, Am. J. Phys. 47, 264 (1979).

[2] G. A. Siviloglou and D. N. Christodoulides, Opt. Lett. 32, 979 (2007).

[3] J. Baumgartl, M. Mazilu, and K. Dholakia, Nat. Photonics 2, 675 (2008).

[4] P. Polynkin, M. Kolesik, J. V. Moloney, G. A. Siviloglou, and D. N. Christodoulides, Science 324, 229 (2009).

[5] P. Rose, F. Diebel, M. Boguslawski, and C. Denz, Appl. Phys. Lett. 102, 101101 (2013).

[6] N. Wiersma, N. Marsal, M. Sciamanna, and D. Wolfersberger, Opt. Lett. 39, 5997 (2014).
[7] T. Vettenburg, H. I. Dalgarno, J. Nylk, C. Coll-Lladó, D. E. Ferrier, T. Čižmár, F. J. Gunn-Moore, and K. Dholakia, Nat. Methods 11, 541 (2014).

[8] A. Lotti, D. Faccio, A. Couairon, D. G. Papazoglou, P. Panagiotopoulos, D. Abdollahpour, and S. Tzortzakis, Phys. Rev. A 84, 021807(R) (2011).

[9] P. Panagiotopoulos, D. Abdollahpour, A. Lotti, A. Couairon, D. Faccio, D. G. Papazoglou, and S. Tzortzakis, Phys. Rev. A 86, 013842 (2012).

[10] I. Kaminer, M. Segev, and D. N. Christodoulides, Phys. Rev. Lett. 106, 213903 (2011). 
[11] Y.Zhang, M. Belić, Z. Wu, H. Zheng, K. Lu, Y. Li, and Y. Zhang, Opt. Lett. 38, 4585 (2013).

[12] N. Wiersma, N. Marsal, M. Sciamanna, and D. Wolfersberger, Sci. Rep. 5, 13463 (2015).

[13] Y. Hu, Z. Sun, D. Bongiovanni, D. Song, C. Lou, J. Xu, Z. Chen, and R. Morandotti, Opt. Lett. 37, 3201 (2012).

[14] N. Wiersma, N. Marsal, M. Sciamanna, and D. Wolfersberger, Sci. Rep. 6, 35078 (2016).

[15] R. Y. Chiao, E. Garmire, and C. H. Townes, Phys. Rev. Lett. 13, 479 (1964).

[16] Z. Chen, M. Segev, and D. N. Christodoulides, Rep. Prog. Phys. 75, 086401 (2012).
[17] E. DelRe and M. Segev, Self-Focusing and Solitons in Photorefractive Media (Springer, New York, 2009), pp. 547-572.

[18] K. Kos, H. Meng, G. Salamo, M.-f. Shih, M. Segev, and G. C. Valley, Phys. Rev. E 53, R4330(R) (1996).

[19] M. Belić, P. Jander, K. Motzek, A. Desyatnikov, D. Jović, A. Strinić, M. Petrović, C. Denz, and F. Kaiser, J. Opt. B: Quantum Semiclassical Opt. 6, S190 (2004).

[20] L. Allen, M. Padgett, and M. Babiker, Prog. Opt. 39, 291 (1999).

[21] D. Faccio, A. Lotti, A. Matijosius, F. Bragheri, V. Degiorgio, A. Couairon, and P. Di Trapani, Opt. Express 17, 8193 (2009). 